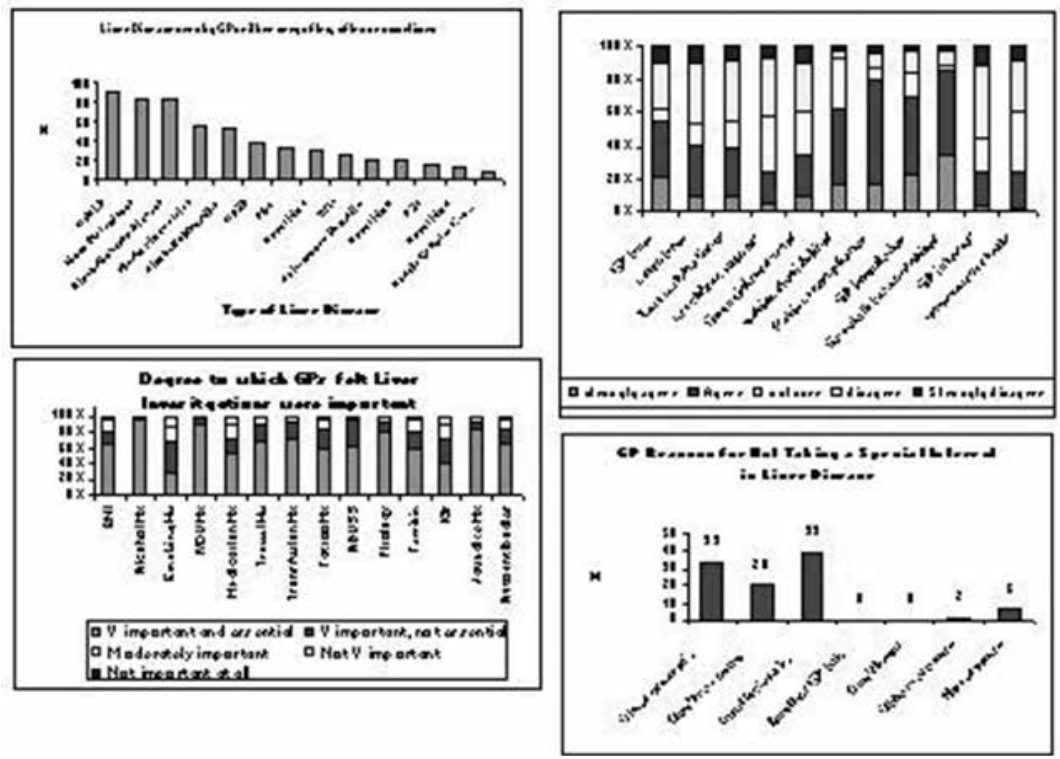

Abstract PTU-098 Figure

Graph 2 - The degree to which GPs felt liver investigations were important in the clinical management of liver disease in primary care.

For investigations that GPs graded as 'Very Important and Essential' many were not, in 'real-life' primary care practise, documented in patient records (Williams et. al. 2012).

Graph 3 - The degree to which potential barriers might influence GP capacity to manage liver disease in primary care.

Only $6 \%$ of GPs stated they had a 'special interest' in liver disease and no GP stated that someone else in the practise took the lead on liver disease.

Graph 4 - Reasons for GPs not having a 'special interest' in liver disease

However, as 'generalists', 83\% of GPs felt they needed more educational support via protected learning sessions, improved national guidelines and joint specialist-GP clinics.

Conclusion This survey revealed that many GPs, despite not having a 'special interest' in liver disease, would welcome greater educational support from specialists and improved national guidelines. The barriers most cited as influencing GP capacity to manage liver disease are surmountable and should be the focal point for new integrated care pathways.

Disclosure of Interest None Declared

\section{PTU-099 DISCOVERY OF POTENTIAL PLASMA BIOMARKERS OF CHOLANGIOCARCINOMA UTILISING SURFACE-ENHANCED LASER DESORPTION/IONIZATION TIME-OF-FLIGHT MASS SPECTROMETRY (SELDI-TOF MS)}

doi:10.1136/gutjnl-2013-304907.189

1."C A Wadsworth, 2V M Horneffer-van der Sluis, 'A Zabron, 'S D Taylor-Robinson, 'R J Edwards, 'S A Khan. 'Department of Hepatology and Gastroenterology; ${ }^{2}$ Centre of Pharmacology and Therapeutics, Imperial College London, London, UK

Introduction Cholangiocarcinoma (CC) is a malignant neoplasm of the bile duct. Diagnosis of CC is hampered by the inadequate performance of current plasma markers of disease, particularly in patients with preexisting primary sclerosing cholangitis (PSC). We aimed to identify potential new protein biomarkers of CC

Methods In an initial discovery study, blood plasma samples from 18 subjects with CC, 17 with PSC and 10 healthy controls were subjected to SELDI-TOF MS. Comparisons of $\mathrm{m} / \mathrm{z}$ peak intensity were made between groups using the Mann-Whitney $U$ test. Differentiating $\mathrm{m} / \mathrm{z}$ peaks were then confirmed in a further validation study of 81 subjects with CC, 54 with PSC and 90 healthy controls. Pearson's correlation was used to investigate the relationship of each $\mathrm{m} / \mathrm{z}$ peak's intensity to routine laboratory indices. Diagnostic performance was investigated using receiver operator characteristic area-under-the curve (ROC-AUC) analyses. Multiple linear regression was used to investigate the performance of combinations of differentiating $\mathrm{m} / \mathrm{z}$ peaks, as well as the combination of $\mathrm{m} / \mathrm{z}$ peaks with routine laboratory markers (including CA19-9).

Results Seven differentially expressed $\mathrm{m} / \mathrm{z}$ peaks were identified in the CC group and these were subsequently confirmed in the validation study $(p=2.6 \times 10-4$ to $9.4 \times 10-13)$. The intensity of the seven $\mathrm{m} / \mathrm{z}$ peaks of interest did not correlate with creatinine, ALP, bilirubin, CRP, white cell count or CA19-9. A panel of three peaks discriminated CC from PSC subjects with ROC-AUC of 0.76 (sensitivity $75 \%$, specificity $64 \%$ ). A panel of five peaks discriminated CC subjects from healthy controls with ROC-AUC of 0.90 (sensitivity $95 \%$, specificity $74 \%$ ). Addition of routine laboratory indices did not change the diagnostic performance of these models significantly.

Conclusion SELDI-TOF has been used to successfully identify seven $\mathrm{m} / \mathrm{z}$ peaks that are differentially intense in CC subjects (total $\mathrm{n}=99)$, when compared to PSC subjects $(\mathrm{n}=64)$ and healthy controls $(n=107)$. These peaks appear to be independent of standard markers of renal impairment, cholestasis, sepsis and inflammation, as well as CA19-9. Individually, and more so in combination, these peaks exceed the expected diagnostic performance of CA19-9, particularly in discriminating CC from PSC. Work to identify the proteins represented by these $\mathrm{m} / \mathrm{z}$ peaks is ongoing.

Disclosure of Interest None Declared

\section{PTU-100 DECOMPENSATED ALCOHOLIC LIVER DISEASE (ALD) IS ASSOCIATED WITH STARTING HEAVY DRINKING AT AN OLDER AGE: A CASE-CONTROL STUDY}

doi:10.1136/gutjnl-2013-304907.190

1."D Gleeson, 'A K Ali, ' J S Jones, 'M Bradley, ${ }^{2} \mathrm{R}$ J Peck, ${ }^{3} \mathrm{~K}$ M McCormack. 'Liver Unit; ${ }^{2}$ Radiology; ${ }^{3}$ Research, Sheffield Teaching Hospitals, Sheffield, UK 